\title{
NUMERICAL SIMULATION OF A SEPARATING LAMINAR BOUNDARY LAYER EXPOSED TO AMBIENT TURBULENCE
}

\author{
EIKE TANGERMANN ${ }^{1}$ AND MARKUS KLEIN ${ }^{2}$ \\ ${ }^{1}$ Bundeswehr University Munich, 85577 Neubiberg, Germany, eike.tangermann@unibw.de \\ ${ }^{2}$ Bundeswehr University Munich, 85577 Neubiberg, Germany, markus.klein@ unibw.de
}

Key words: Laminar Separation, Transition, Hybrid RANS-LES, Synthetic Turbulence

\begin{abstract}
Laminar separation on wings exposed to flow of a low Reynolds number is a very common phenomenon already at low angles of attack. It is extremely sensitive to turbulence in the freestream as is can affect the boundary layer upstream of the separation, the transition into turbulence and at very large length scales also locally change the angle of attack. Hybrid RANS/LES simulations of laminar separation haven been performed with parametric variations of both intensity and length scale of the ambient turbulence. The boundary layer has then been analyzed in order to demonstrate the effect of eddies impinging the laminar boundary layer upstream of the separation leading to stabilizing and destabilizing effects, which both have been identified and visualized.
\end{abstract}

\section{INTRODUCTION}

The boundary layer on a wing operated at low Reynolds numbers is likely to separate before it experiences transition to turbulence. Depending on the airfoil geometry, this occurs already at low angles of attack. Following the separation, Kelvin-Helmholtz vortices are formed above the separated layer, which break up into turbulence and hence the flow re-attaches forming a turbulent boundary layer. This process is very sensitive to ambient turbulence impinging the boundary layer upstream of the separation and thereby delaying or accelerating the separation process. Further, the breakup of shear layer vortices is accelerated by local variations introduced by the ambient turbulence.

Istvan and Yarusevich ${ }^{1}$ have performed experiments on a NACA0018 wing in well defined freestream turbulence. The setup of the present study is closely following their design. In previous work ${ }^{2,3}$ the authors have already demonstrated this setup of hybrid RANS-LES simulations with synthetic turbulence introduced upstream of the wing. It could be shown quantitatively, how the probability of local flow separation is affected by ambient turbulence.

In the present paper the focus is set on the investigation of boundary layer properties like thickness and shape factor in order to show the effect of freestream eddies impinging into the boundary layer. Data from numerical simulations using a hybrid RANS-LES approach of two different levels of intensity and three length scales of ambient turbulence has been evaluated with respect to boundary layer properties. Further, individual events of fluctuations impinging into the boundary layer upstream of the separation have been identified and their effect will be discussed. 


\section{SIMULATION SETUP}

\subsection{Airfoil Configuration}

The configuration of a NACA0018 airfoil is closely related to the experimental setup by Istvan et al. ${ }^{1}$ It features a planar, quasi two-dimensional wing section. In span-wise direction periodic boundary conditions have been applied. The ratio between wing span $b$ and chord $c$ is 0.5 . This allows to perform simulations of turbulent fluctuations with a length scale of up to $20 \%$ chord length without the risk of being influenced by the periodic conditions.

Based on the chord length and freestream velocity the Reynolds number is $R e=80000$. At these conditions the flow starts separating at very low angles of attack. Here, an angle of attack of only $\alpha=4^{\circ}$ has been selected. The separation can be expected at approximately one third of the chord length, which gives enough opportunity to observe the attached region under ambient turbulence conditions. When further increasing the angle of attack, the separation would occur far earlier.

Besides the case of zero ambient turbulence, six different turbulent cases have been simulated. They feature two levels of fluctuation intensity $T u$ each at three different length scales $L_{t}$. Table 1 gives an overview of the cases.
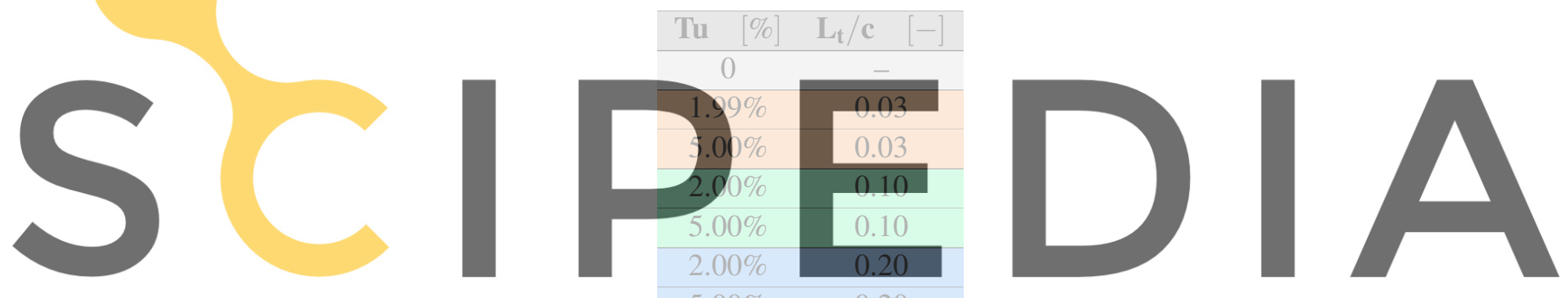

Register for free at https//www.scipedia.com to download the version without the watermark

\subsection{Numerical Setup}

Since the goal is the investigation of turbulent events, it obviously is necessary to resolve turbulent fluctuations at least partially. The phenomena of focus appear close to a wall, therefore DNS and LES both would require a very fine resolution of relatively small structures up to the wall. Both might be affordable in this context for single selected cases only. However, with an increasing number of cases due to targeting a greater range of parameters, these methods become too computaionally expensive. Particularly the larger ambient fluctuations, which come along with larger time scales, require longer physical time spans to build statistics. Therefore, a hybrid RANS-LES approach has been selected. The k $\omega S S T$ based DDES model $^{4,5}$ has been selected. As described in previous work, ${ }^{2}$ the model has to be suppressed upstream of the separation, which has been realized by applying the $\gamma-R e_{\theta}$ criterion, ${ }^{6}$ which features further two transport equations.

All simulations have been carried out using a solver from the OpenFOAM toolbox version 1812. The filtered or averaged Navier-Stokes equations are solved in an incompressible formulation. Pressure coupling is achieved using PISO time stepping together with SIMPLE inner iterations. Time stepping is 

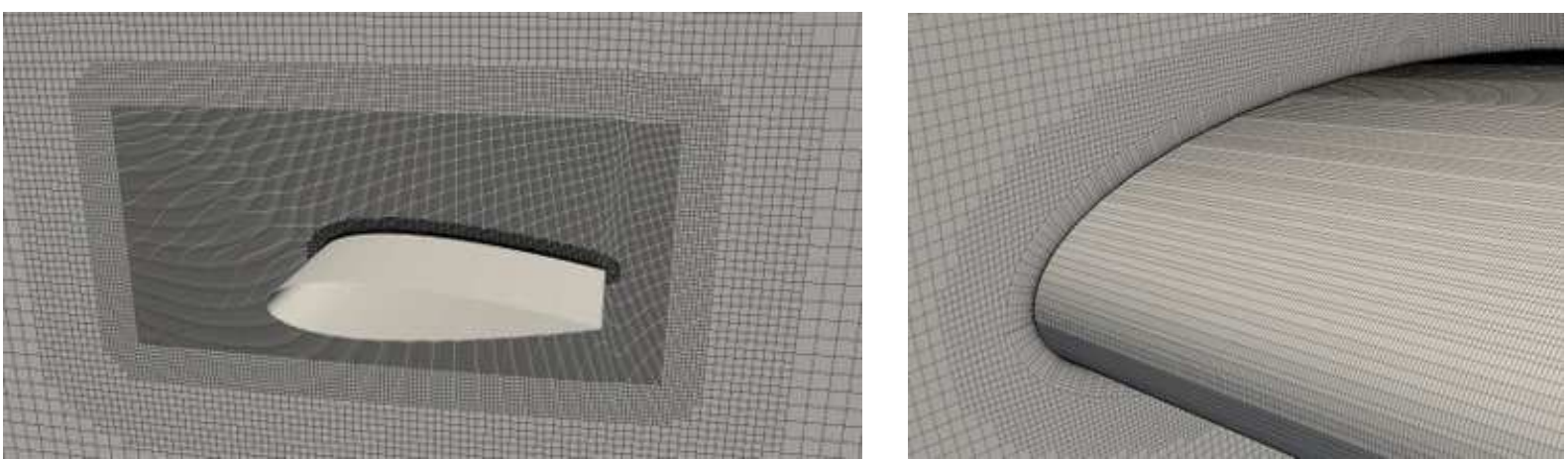

Figure 1: Hierarchically refined structure of the mesh (left) and mesh details around the wing (right).

of second order accuracy using a backward scheme. Spatial discretization also is of second order. The convective terms of the momentum equation are built from central differences blended with $25 \%$ second order upwind for increased stability. In the turbulence model transport equations, the convective terms are stabilized using a TVD scheme.

The mesh is of unstructured topology, based on hexahedra with hierarchical refinement. Instead of hanging nodes the interfaces between refinement layers are fitted with polyhedral cells. For proper resolution of the wall normal direction cell layers are located around the wing. The mesh structure and details of the wing surface are shown in Figure 1. The outer farfield boundary is located 20 times the chord length distant from the wing, which is fa ations from the turbulent region.

2.3 Synthetic Turbulence
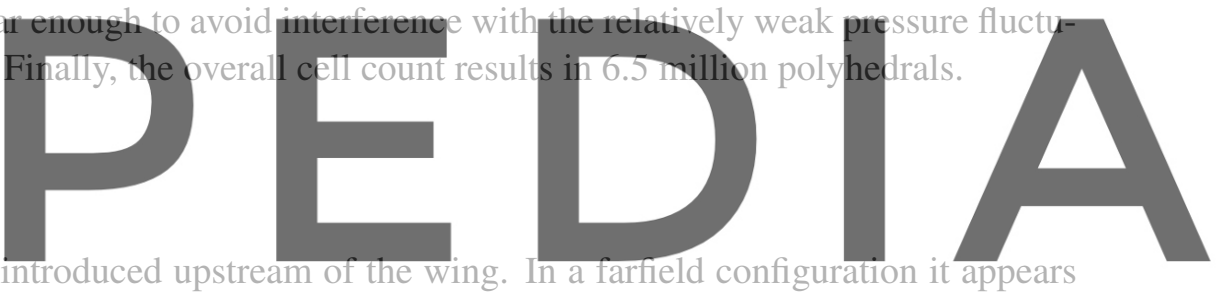

Ambient turbulence needs to be introduced upstream of the wing. In a farfield configuration it appears unfeasible to introduce fluctuations as a boundary condition and have them transported convectively to



computational domain by locally and temporally varying volume forces. The method has been described in detail in Reference.

A force term $\vec{F}^{s y n}$ is added to the momentum equation.

$$
\frac{\partial \vec{u}}{\partial t}+\nabla \cdot(\vec{u} \vec{u})-\nabla \cdot(v \nabla \vec{u})=-\nabla p+\vec{F}^{s y n}
$$

The force represents an acceleration of a fluid element while it is crossing the forcing zone. Modifying the momentum equation has an impact to the pressure correction equation, where the divergence of the force term appears.

$$
\nabla\left(\frac{1}{a_{p}} \nabla p\right)=\nabla\left(\frac{H(\vec{u})}{a_{p}}\right)+\nabla \vec{F}^{s y n}
$$

For an exact result the force field would have to be moved along with the flow while passing the forcing zone. In order to simplify the implementation, instead one slice from the fluctuation field is spread along the forcing zone for each time step. This procedure introduces a dependence of the resulting fluctuation intensity on the length of the forcing region. To compensate for the effect, a control loop featuring 


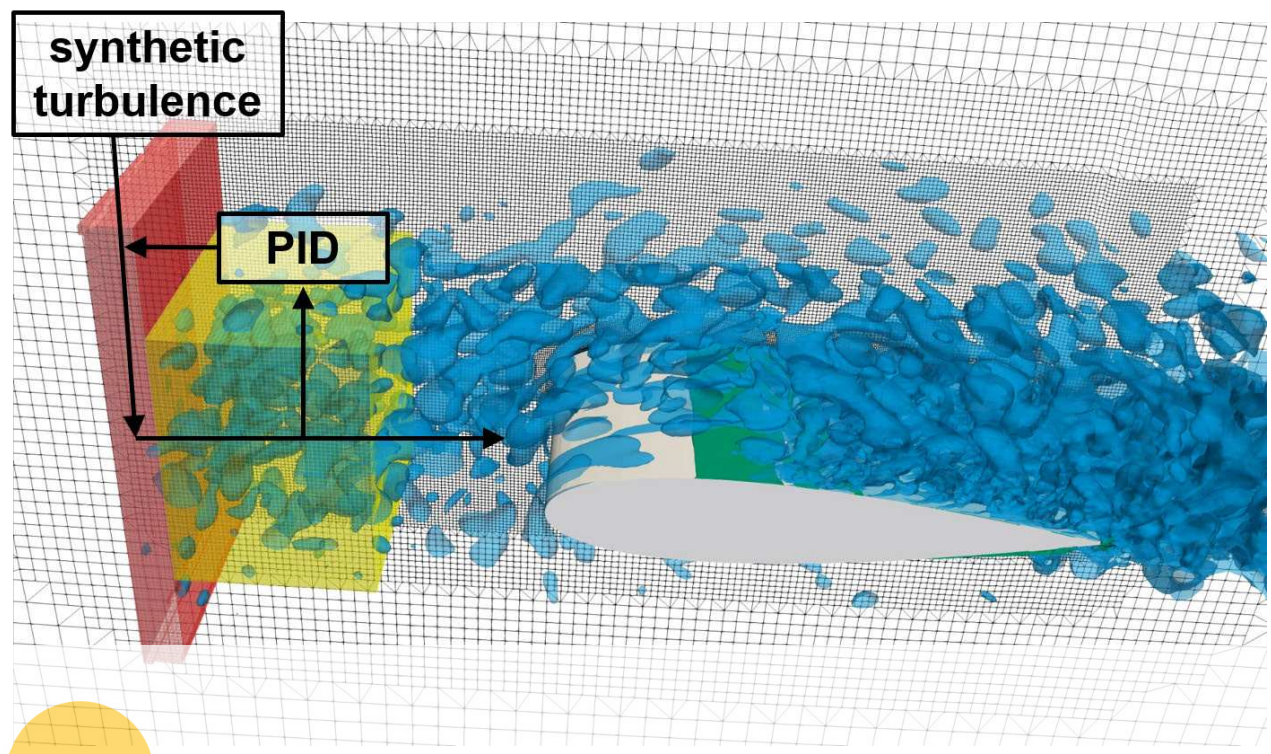

Figure 2: PID control loop with forcing zone (red) and measurement zone (yellow) located upstream of the wing.
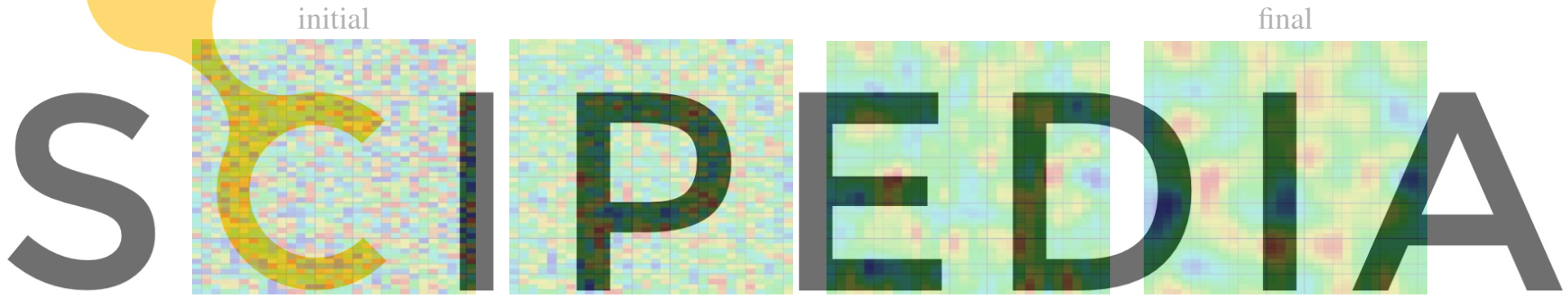

Figure 3: Development of synthetic fluctuations by diffusive filtering process

Register for free at https//www.scipedia.com to download the version without the watermark

a PID controller has been applied, which measures the resulting fluctuation intensity and scales the forcing accordingly. The controller coefficients are determined from delay and rise times from convective transport. Their calibration has been described in Reference. ${ }^{7}$

The overall setup is shown in Figure 2 with the forcing zone marked red located approximately $0.75 c$ upstream of the leading edge followed immediately by the measurement zone marked yellow. It also can be seen how the mesh is refined within the turbulent region. For an accurate transport of the fluctuations the refinement is required from the forcing region downstream.

The length scale of the fluctuations is defined within the generation of the synthetic fluctuation field, which follows the procedure described by Kempf et al. ${ }^{8}$ of filtering a randomly fluctuating field by applying a specific amount of diffusion until the desired length scale is achieved as illustrated $n$ Figure 3. Subsequently, the field is transformed to fulfill the prescribed Reynolds stresses. 


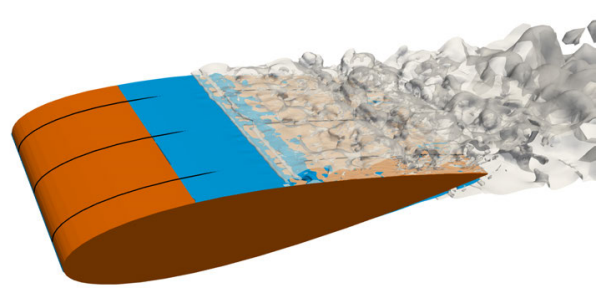

$T u=0$

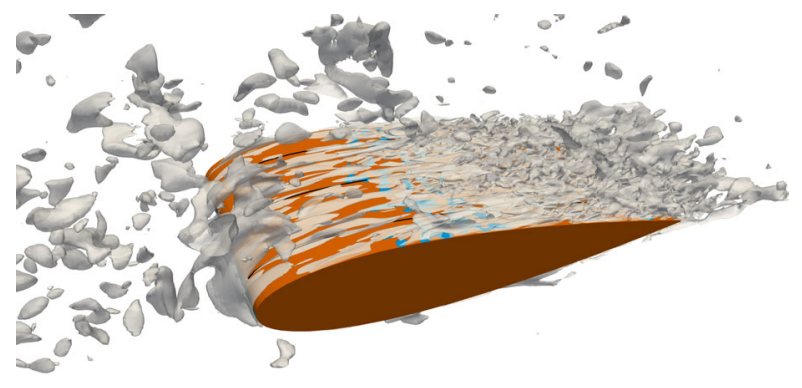

$T u=5 \%, L_{t}=0.03 c$
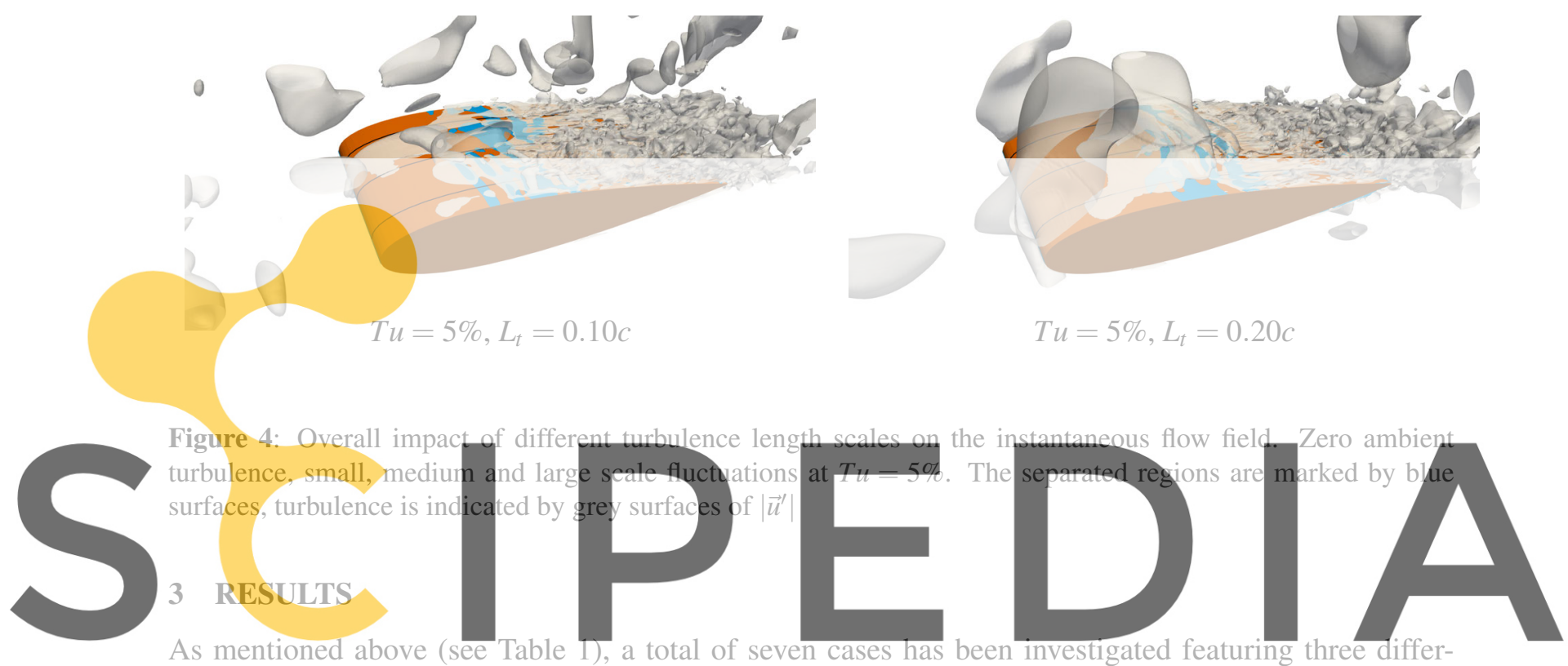

As mentioned above (see Table 1), a total of sevent cases has been investigated featuring three differ-

ent fluctuation length scales and two levels of turbulence intensity. The overall dynamic of the process

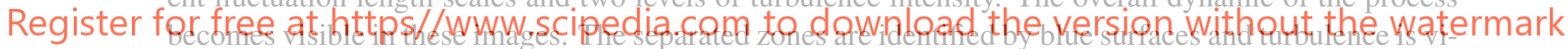

sualized by grey iso-surfaces of instantaneous $\left|\vec{u}^{\prime}\right|$. As expected the zero turbulence case shows a straight

line of initiai separation. Shear layer vortices are aiso formed in a straight line. As they break up into turbulence the flow field becomes less regular and the flow re-attaches. Only small spots of separated areas appear occasionally along the turbulent part of the boundary layer.

In the cases with ambient turbulence the separation zone appears very different. In the small scale case only isolated patches of separated flow occur within the region where previously full separation had appeared. At increased fluctuation length scale the separated regions become more contiguous and form lines approximately in span-wise direction.

It needs to be noted that the boundary layer still is of laminar type even though ambient turbulence impinges on the wing surface. When the fluctuations hit the wall, they get stretched in stream-wise direction as they start to enter the slower flow within the boundary layer while the ambient flow continues its convective transport. This results in a laminar boundary layer with local variations of the velocity. These variations lead to changes in the separation behaviour. Downstream of the separation zone the boundary layer actually has become turbulent, which is impressively and clearly visible in Figure 4.

It should be noted that the turbulent transition does not take place within the boundary layer. Instead, 


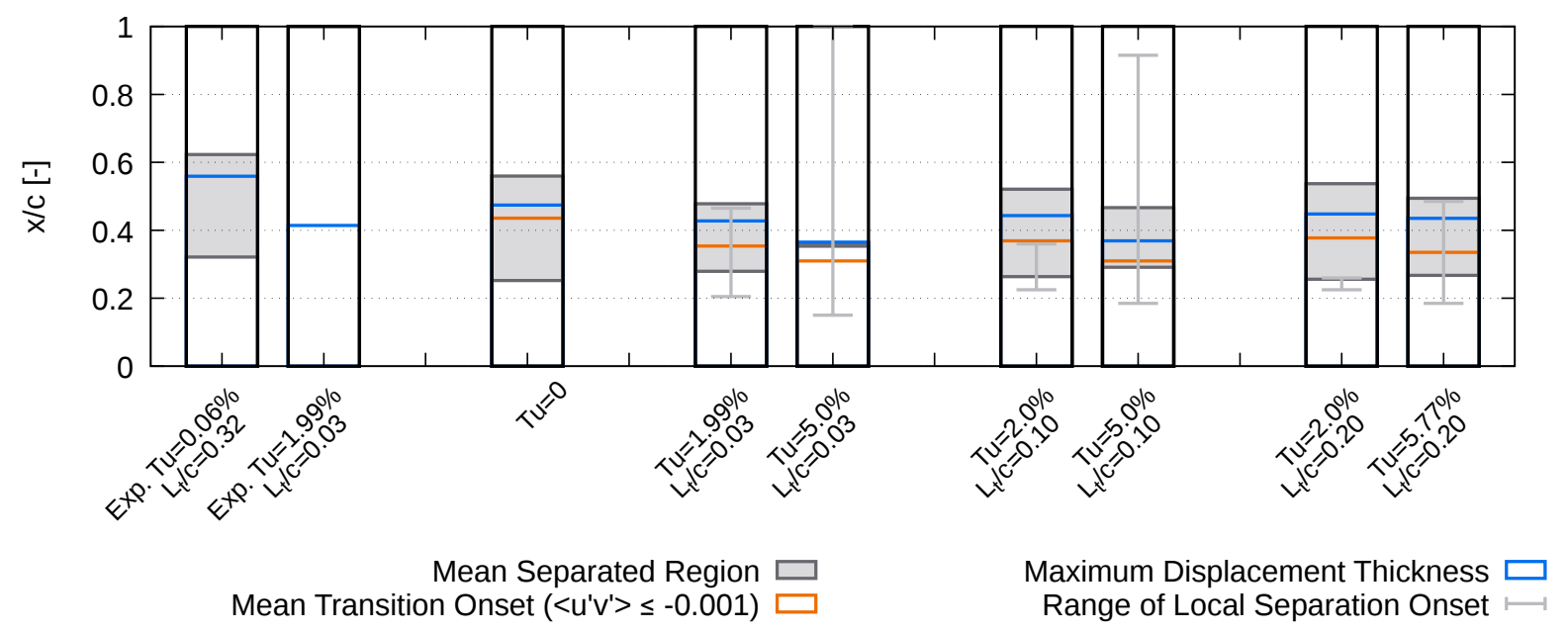

Figure 5: Locations of separation, transition and re-attachment based on evaluation of the averaged flow field. Experimental data presented for comparison from Istvan et al. ${ }^{1}$

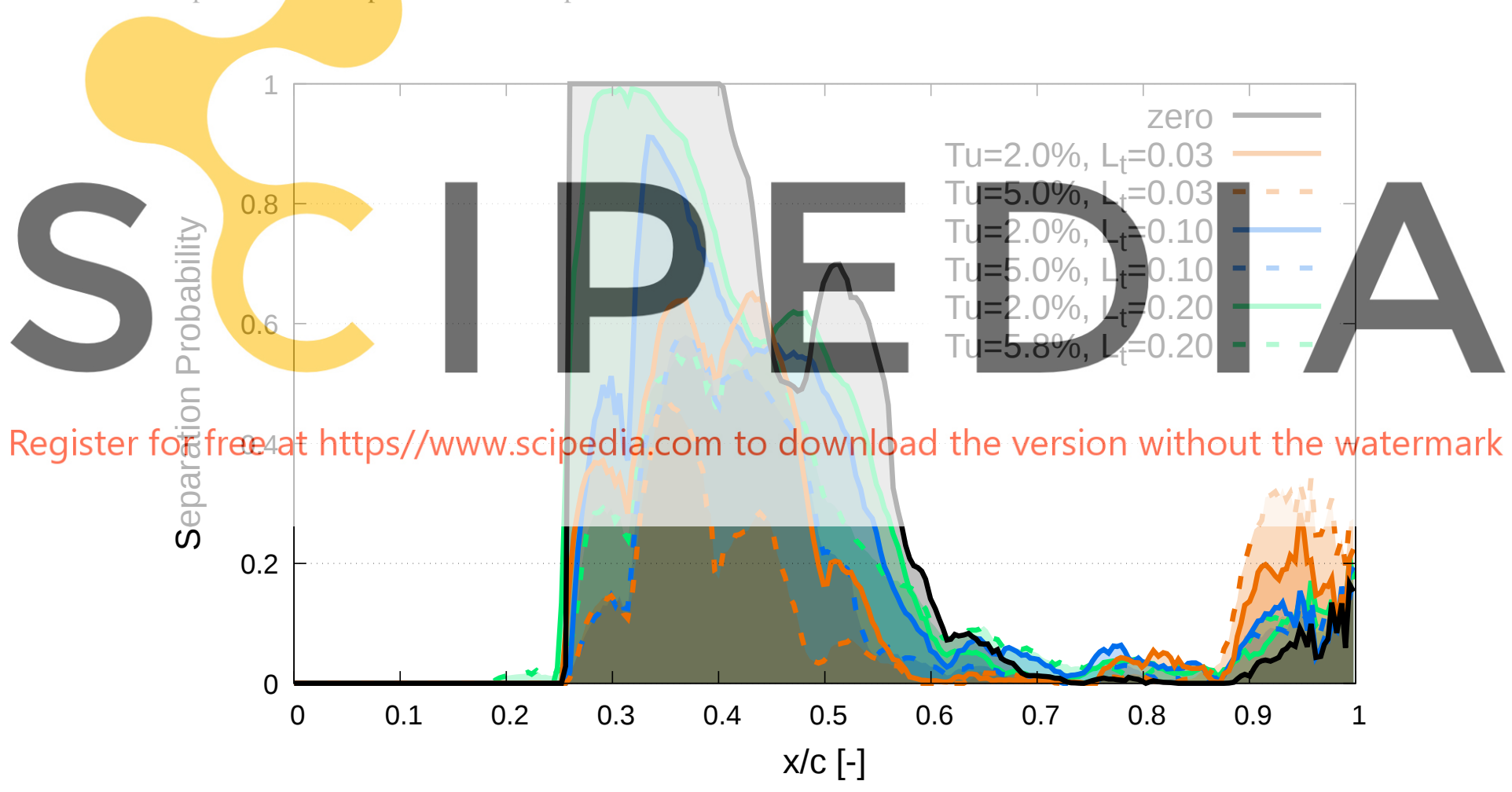

Figure 6: Probability of separation along the upper side of the wing.

it occurs above the separated regions. There, the DDES turbulence model is in the LES mode. For this reason it is capable of producing resolved turbulence during the transition process.

Figure 5 shows the locations of separation, transition and re-attachment resulting from the averaged flow field. For comparison also experimental data from Istvan et al. ${ }^{1}$ is shown. In the experimental data 

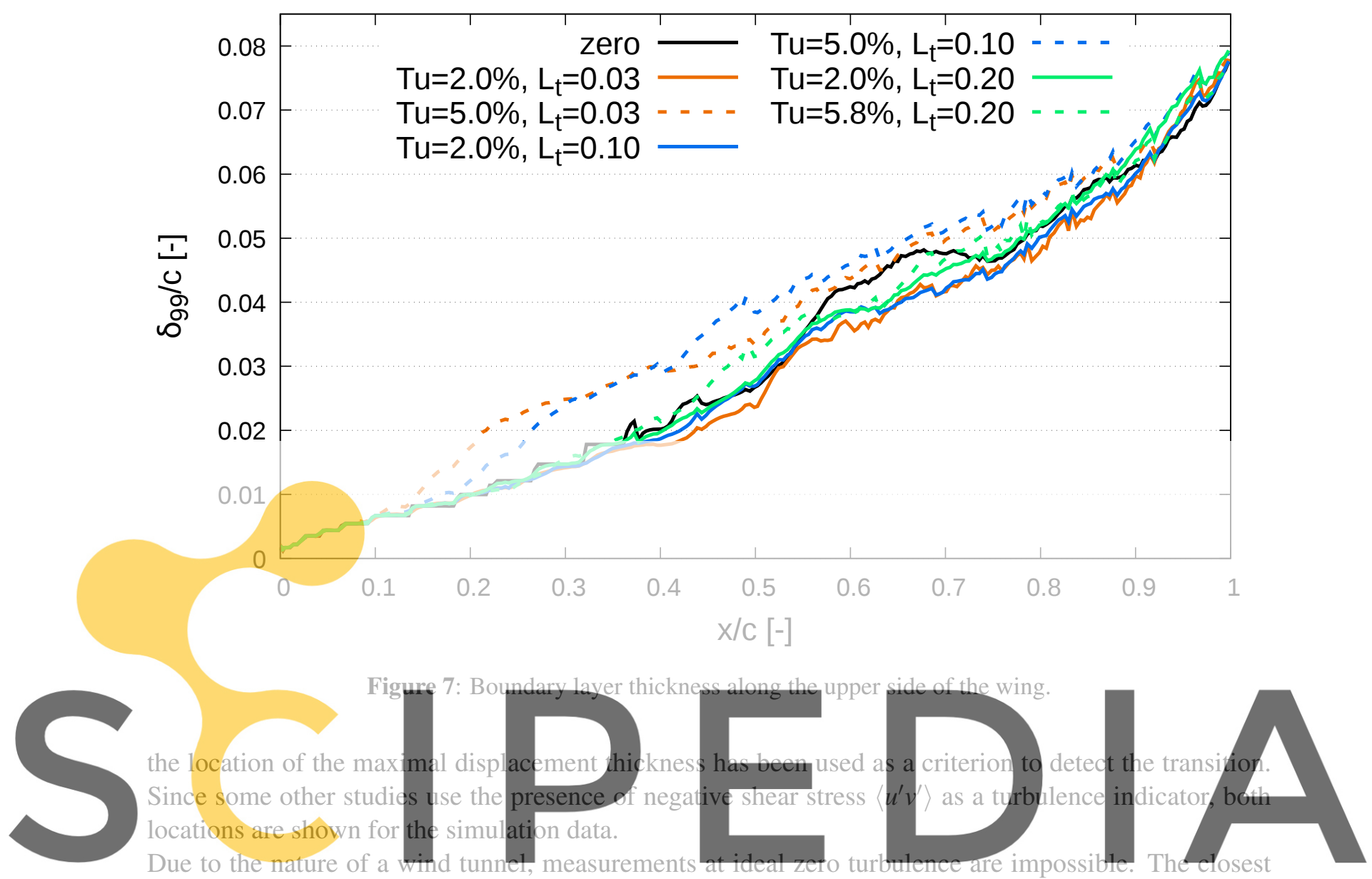

state shown by Istvan et al. is at an intensity of $T u=0.06 \%$ and at a very large length scale. When

Register fornf

flow separation. The length of the mean separated region is very similar. Within the separated region the

transition seems to be produced slightly sooner in the simulation.

In ambient turbulence of $T u=1.99 \%$ the experiment does not show a separation in the mean flow field, whereas the simulation still produces a significant separated region. It also can be seen that the onset of the separated region undergoes strong variations as denoted by the error bars. They indicate the most upstream and most downsteam locations observed during the investigation time along stream-wise lines on the surface.

When increasing the turbulence intensity to $5 \%$, the mean separation also vanishes almost completely in the simulation. But still separated areas are present in the instantaneous field. The stream-wise variation of the separation onset ranges from even further upstream than in the zero turbulence case up to no separation along the line. There is still a clear transition onset since, as mentioned before, the transition takes place distant from the wall above the separated spots. At medium and large scale turbulence the separated regions clearly appear for both settings of intensity. It shows, that the larger fluctuations cannot affect the boundary layer as strong as the small fluctuations.

Since it has been shown that separation even occurs if it is not visible in the mean flow field, the prob- 

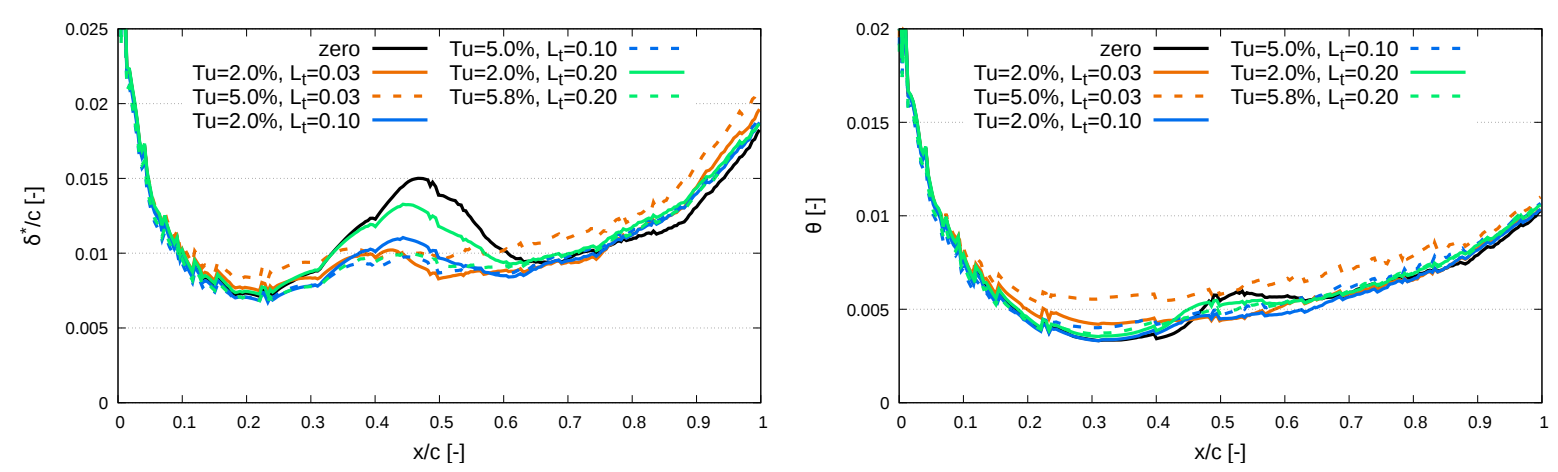

$\mathrm{x} / \mathrm{c}[-1$
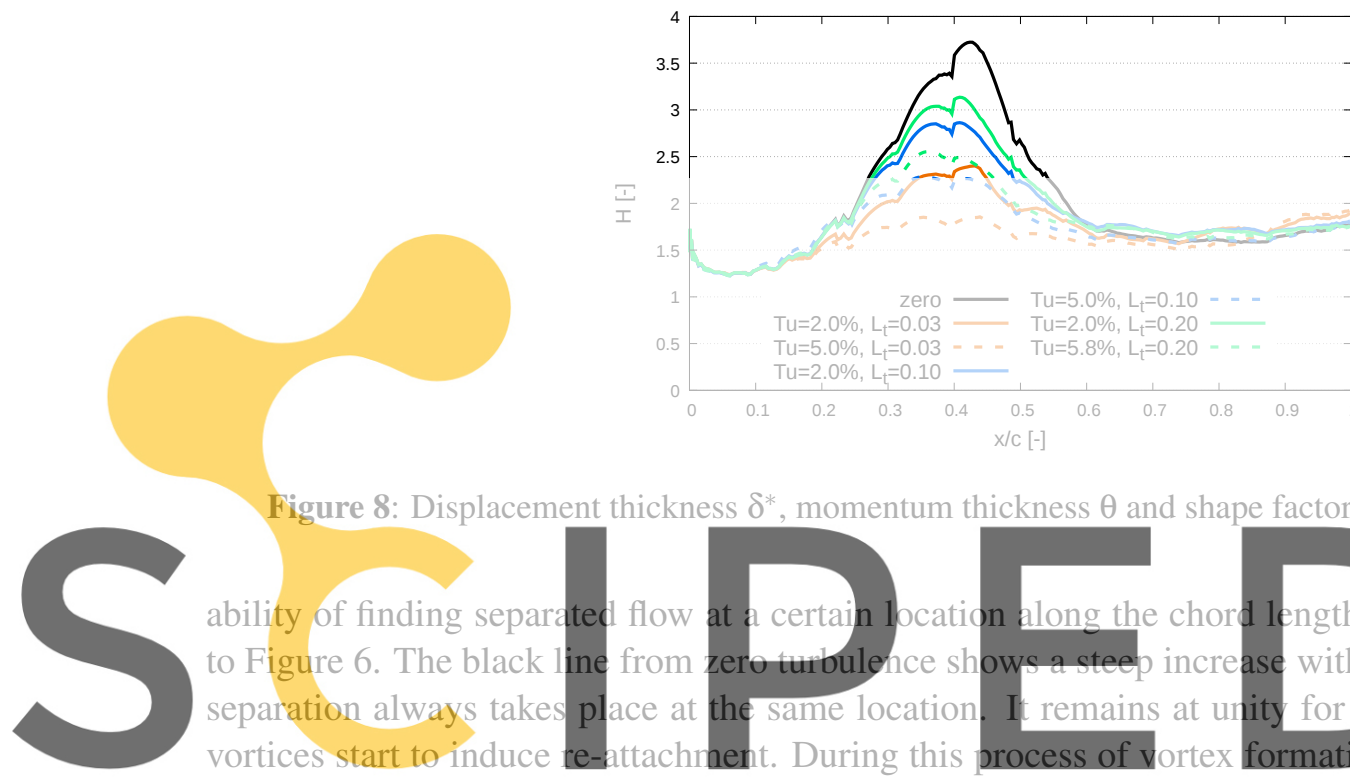

Figure 8: Displacement thickness $\delta^{*}$, momentum thickness $\theta$ and shape factor $H$ along the wing surface

ability of finding separated flow

to Figure 6. The black line

separation always takes

vortices start to induce

peak of separated flow occurs. This happens during the breakup process.

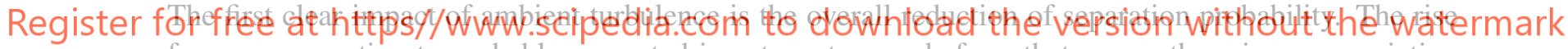

from no separation to probably separated is not as steep as before, that means there is some variation

in the separation location. It should be noted that an additional wavy pattern becomes visible, which

is identical for all curves. This is attributed to inaccuracies in the surface curvature and is not directly related with the investigated phenomena. However, it strongly underlines the sensitivity of the process to the geometry and demonstrates the importance to take deviations into consideration.

Generally, the probability plot shows that by increased turbulence intensity, which is associated with the dashed lines, unsurprisingly the flow tends to show less separation. But also a strong influence of the length scale can be seen. The solid green curve for large scale, low intensity shows a behaviour quite similar to the zero turbulence case. The rise is not as steep and the transition and re-attachment occur slightly earlier. In contrast the solid orange curve from small scale, low intensity shows a strongly decreased separation probability, and further, it does not feature the secondary probability peak during the transition process seen before.

The small and medium scale fluctuations only lead to a delay of separation. The large scale fluctuations, however, also show a slight trend to accelerated separation as the green lines indicate a very slight probability upstream of all other cases. 

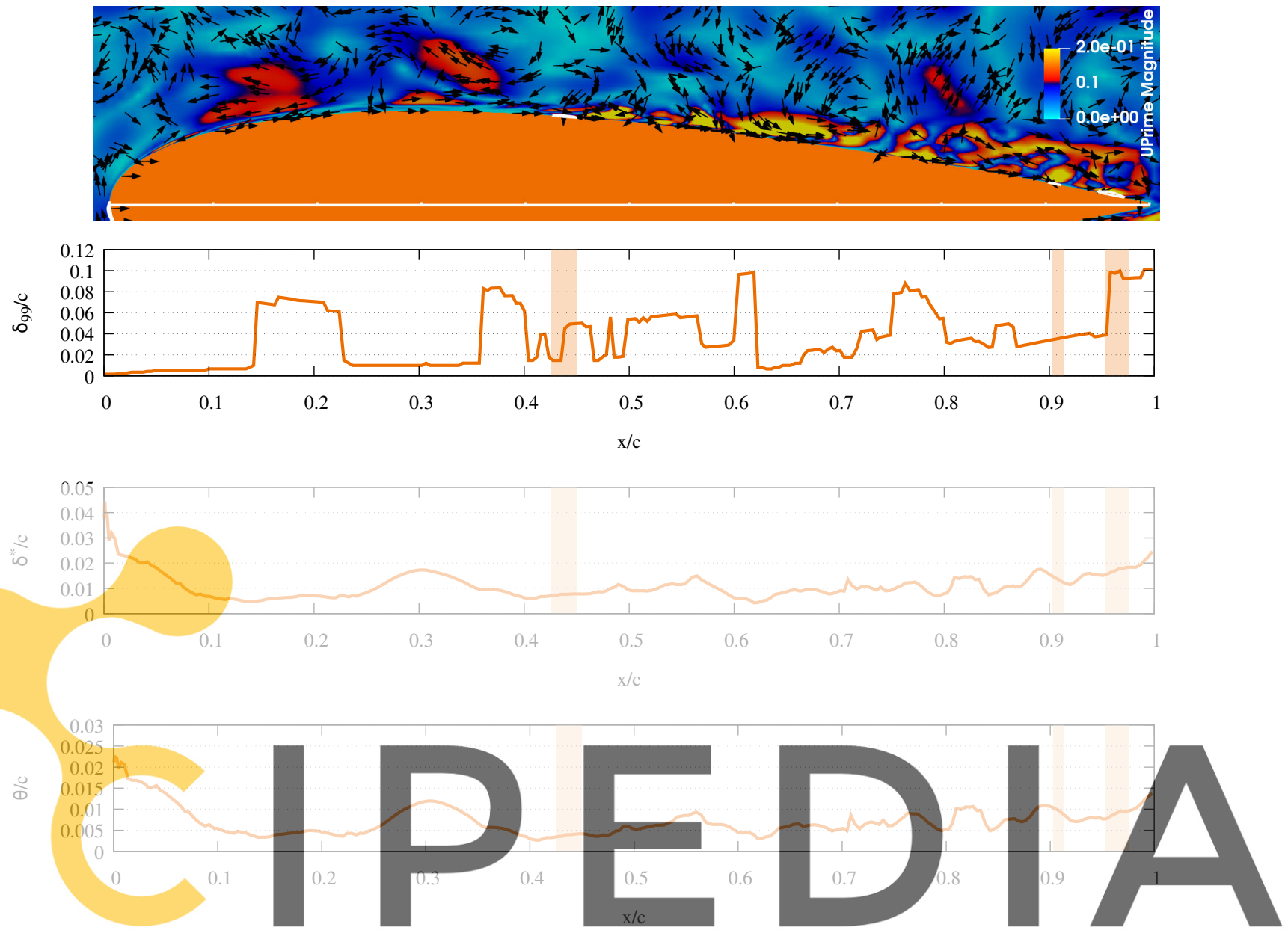

Register for fręe at https//www.scipedia.com to download the version without the watermark

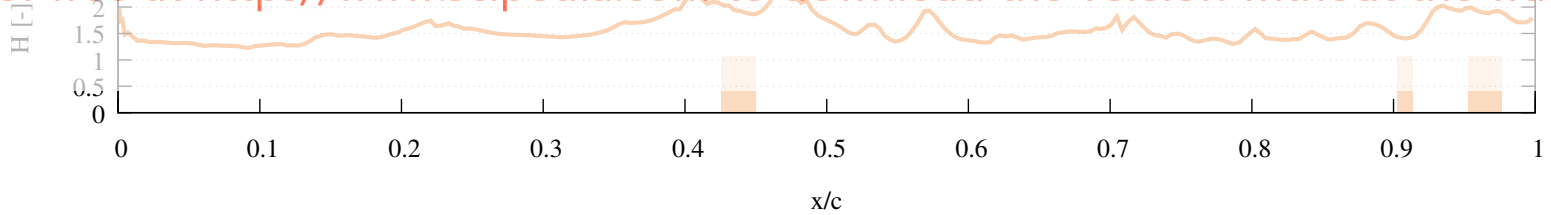

Figure 9: First instantaneous view for small scale high intensity fluctuations. The contour plot shows the magnitude of deviation from local mean velocity together with their vectors to indicate the direction of the fluctuations. White isolines in the contour plot and orange bars in the line plots indicate separated regions. Decelerating eddy at $x / c=0.2$, accelerating eddy at $x / c=0.3$.

The separation behaviour indicates that the small scale fluctuations have a stronger impact to the boundary layer as they produce stronger spatial gradients since each turbulent event acts on a smaller spot. In Figure 7 the boundary layer thickness in terms of $\delta_{99}$ is shown. In order to take the influence of events associated with the ambient turbulence into account, it is insufficient to analyse the mean flow field. It rather is necessary to calculate the boundary layer thickness for instantaneous data, which shows indi- 

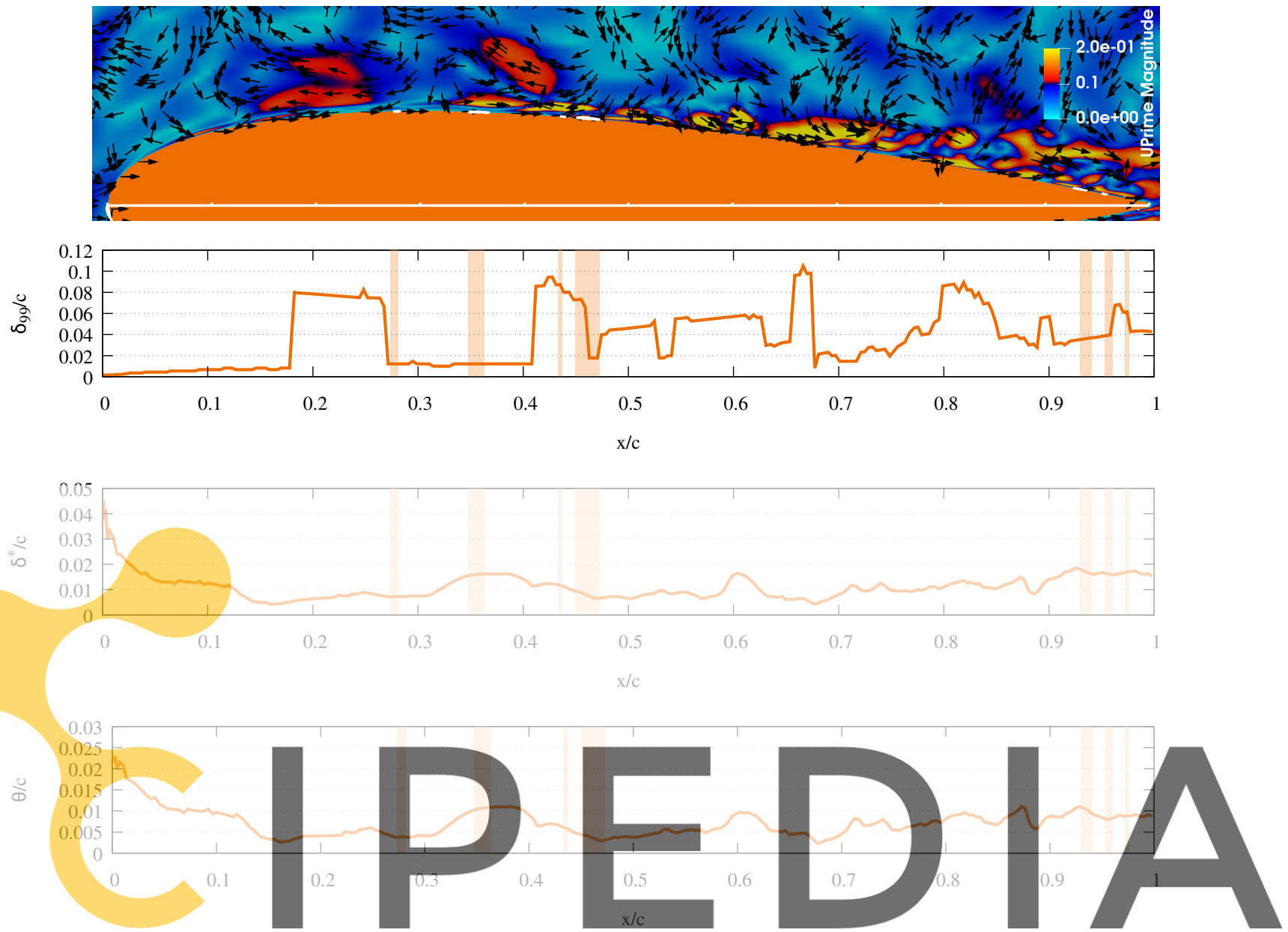

Register for fręe at https//www.scipedia.com to download the version without the watermark

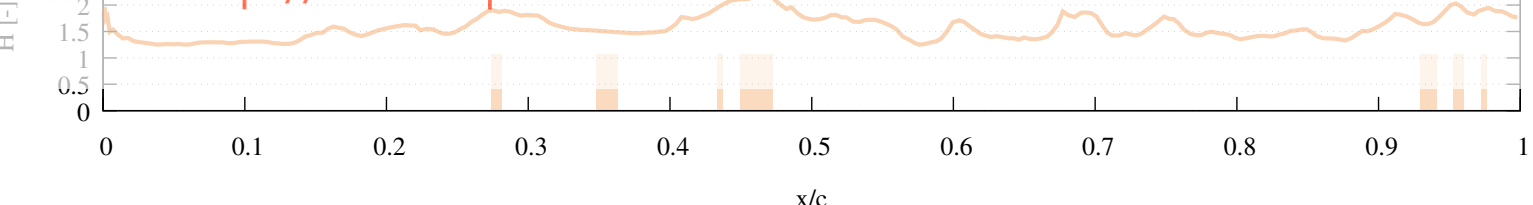

Figure 10: First instantaneous view, same schematic as above. Onset of separation at $x / c=0.25$.

vidual events. Data from many instants has then been averaged to the data plotted in Figure 7.

In terms of $\delta_{99}$ thickness the influence of turbulence only becomes visible for the high intensity cases. Small and medium scale fluctuations lead to a clear increase of the boundary layer thickness soon after the leading edge. The boundary layer then stays thicker until shortly before the trailing edge. A slight thickening also seems to appear in the large scale case but it starts only after reaching the separation zone.

The behaviour of the $\delta_{99}$ thickness shows the general trend of a boundary thickening by the ambient turbulence. Its impact on separation can better be seen from displacement thickness $\delta^{*}$ and momentum 
thickness $\theta$ and their ratio, the shape factor $H$. All of them are shown in Figure 8 along the upper side of the wing. The small scale turbulence produces higher values for $\delta^{*}$ and $\theta$, which is clearly seen for high intensity and only slightly for low intensity. Medium and large scale fluctuations have almost no influence upstream of the separation. The shape factor, however, does not show significant differences in the region of attached flow. But it clearly shows differences within the separation zone, where it clearly correlates with the probability of separation. The stronger separation is prevented by the ambient fluctuations the lower is the shape factor. In terms of shape factor the effect of small scale turbulence at low intensity appears as strong as that of medium scale turbulence at high intensity, whereas large scale at low intensity is relatively close to the zero turbulence case.

To understand the processes that occur when a turbulent eddy impinges on the surface, it is useful to investigate individual events. Two instants from the small scale high intensity case are shown in Figures 9 and 10. The contour plot shows the magnitude of fluctuation velocity together with its vectors. This helps to identify the fluctuations. Patches of separated flow are marked with white lines, which appear very close to the surface.

In the first instant (Figure 9) two significant eddies are moving along the surface upstream of the separation zone. Around $x / c=0.2$ a decelerating eddy causes a strong increase of the $\delta_{99}$ thickness. This is related with what could be seen in the averaged plots above. On its downstream side at $x / c=0.25$ already a very slight peak of $H$ can be seen, which indicates that a separation could happen if the effect increases. Around $x / c=0.3$ the opposite is happening. The accelerating eddy increases both $\delta^{*}$ and $\theta$ but the shape factor $H$ even slightly decreases indicating that the boundary layer is stabilized and separation is prevented. Immediately downstream of that event another decelerating eddy is located a little further away from the surface. It also leads to an increase of the shape factor and could very likely cause flow separation soon afterwards. However, then it also enters the turbulent part of the boundary layer, where the eddies become torn apart into boundary layer turbulence, which does not favour separation. The next instant in Figure 10 is slightly later, the structures have traveled approximately $\Delta x / c=0.05$. The first decelerating eddy now extends from $x / c=0.18$ to 0.27 and has lead to the formation of a small separated spot at $x / c=0.28$. This comes together with a further increase of the shape factor.

\section{CONCLUSIONS}

The presented hybrid RANS/LES simulations of laminar separation of flow over a NACA0018 wing in ambient turbulence of different length scales and at different intensities have shown a strong dependence on both of these parameters. Small scale fluctuations can more directly impinge into the boundary layer, affect the boundary layer velocity profiles and thereby its separation behaviour. It has been shown, that small scale fluctuations at lower intensity can even have a higher impact than larger scale fluctuations at higher intensity.

Generally, the ambient turbulence stabilizes the flow and partially prevents it from separation. This can be measured by a decrease of the boundary layer shape factor along the region of separation, which correlates with the reduction of separation probability.

The investigation of individual turbulent events shows that eddies passing along the surface can either stabilize or destabilize the flow depending on their effective direction. However, besides the direct effect on the separation they also accelerate the breakup of shear layer vortices from above separated zones into boundary layer turbulence, which then prevents further separation to a great extent. Therefore, the 
overall contribution of ambient turbulence is a stabilizing effect.

\section{REFERENCES}

${ }^{1}$ Istvan, M.S. and Yarusevych, S. Effects of Free-Stream Turbulence Intensity on Transition in a Laminar Separation Bubble Formed over an Airfoil. Experiments in Fluids (2018) 59:52.

${ }^{2}$ Tangermann, E. and Klein, M. Numerical Simulation of Laminar Separation on a NACA0018 Airfoil in Freestream Turbulence. In AIAA Scitech 2020 Forum. American Institute of Aeronautics and Astronautics, (2020).

${ }^{3}$ Tangermann, E. and Klein, M. Numerical Simulation of Laminar Separation on an Airfoil in SmallScale Freestream Turbulence. In: New Results in Numerical and Experimental Fluid Mechanics XII, Springer (2020) 619-629.

${ }^{4}$ Menter, F. R., Kuntz, M., and Langtry, R., Ten Years of Industrial Experience with the SST Turbulence Model, Turbulence, heat and mass transfer, (2003) 4(1):625-632.

${ }^{5}$ Spalart, P. R., Deck, S., Shur, M. L., Squires, K. D., Strelets, M. K., and Travin, A., A New Version of Detached-eddy Simulation, Resistant to Ambiguous Grid Densities, Theoretical and Computational Fluid Dynamics, (2006) 20(3):181-195.

${ }^{6}$ Langtry, R. B., and Menter, F. R., Correlation-Based Transition Modeling for Unstructured Parallelized Computational Fluid Dynamics Codes, AIAA journal, (2009) 47(12):2894-2906.

${ }^{7}$ Tangermann, E. and Klein, M. Controlled Synthetic Freestream Turbulence Intensity Introduced by a Local Volume Force. Fluids. (2020) 5(3):130.

${ }^{8}$ Kempf, A., Klein, M. and Janicka, J. Efficient generation of initial-and inflow-conditions for transient turbulent flows in arbitrary geometries. Flow, Turbulence and combustion (2005) 74:67-84. 